\title{
THEORETICAL MODELING OF TWO-PHOTON ABSORPTION OF BENZOXAZOLES EXHIBITING EXCITED STATE PROTON TRANSFER
}

\author{
Y. Syetov ${ }^{*}$ \\ Oles Honchar Dnipro National University, Dnipro, Ukraine \\ *e-mail: setov2003@yahoo.com
}

Two-photon absorption spectra of 2-(2'-hydroxyphenyl)benzoxazole, 2,5-bis(2-benzoxazolyl)phenol and 2,5-bis(2-benzoxazolyl)hydroquinone are modeled by time-dependent density functional theory calculations. In contrast to the linear absorption, where the transitions to the two lowest excited states are leading, the two-photon absorption cross sections are significant for the transitions to higher states which are weak in one-photon absorption spectra. The 2,5-bis(2-benzoxazolyl)phenol and 2,5-bis(2benzoxazolyl)hydroquinone demonstrate the maximum cross section of about ten times larger than that of 2-(2'-hydroxyphenyl)benzoxazole. The largest value of the cross section is calculated for 2,5-bis(2benzoxazolyl)hydroquinone to be about 1500 units of Goeppert-Mayer for excitation at $600 \mathbf{~ n m}$. modeling.

Keywords: two-photon absorption, excited state proton transfer, benzoxazole derivatives, molecular

Received 16.11.2018; Received in revised form 18.12.2018; Accepted 21.12.2018

\section{Introduction}

Two-photon absorption (TPA), a simultaneous absorption of two photons, - is a non-linear optical process with intensity depending on the square of incoming light. The TPA process has been gaining greater interest in a number of areas, particularly in the fields of two-photon fluorescent microscopy and imaging, three-dimensional microstructure optical data storage, optical switching, optical power limiting, photodynamic therapy [1]. 2-(2'-hydroxyphenyl)benzoxazole (HBO), 2,5-bis(2benzoxazolyl)phenol (DBP) and 2,5-bis(2-benzoxazolyl)hydroquinone (BBHQ) (Fig. 1) are benzoxazole derivatives with single and double hydrogen bonds undergoing photoinduced excited state intramolecular proton transfer (ESIPT). The ESIPT converts enol structure of the molecules with the $\mathrm{OH}$... $\mathrm{N}$ hydrogen bonds into keto structure with the O...HN hydrogen bonds and causes appearance of fluorescence with an anomalously large Stokes shift. For the compound HBO TPA has been studied in solution in cyclohexane with excitation at wavelength of $532 \mathrm{~nm}$ (photon energy of $2.33 \mathrm{eV}$ ). The value of TPA cross section is estimated to be $9.38 \cdot 10^{-21}$ $\mathrm{cm}^{4} / \mathrm{GW}$ [2]. In this paper we describe a theoretical study on TPA of HBO, DBP and BBHQ aiming evaluation of the substances as efficient media for two-photon absorption.

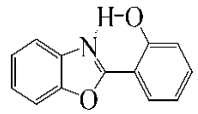

a

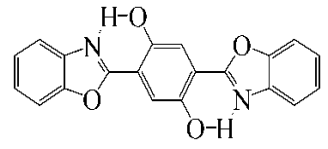

$\mathrm{b}$

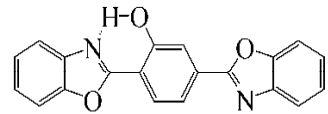

c

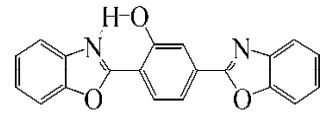

d

Fig. 1. Molecular structure of HBO (a), BBHQ (b) and two conformations of DBP (c, d).

\section{Calculations details}

One-photon absorption (OPA) transitions are described by an oscillator strength given by expression

$$
\delta^{O P A}=\frac{2 \omega_{f}}{3} \sum_{\alpha}\left|\left\langle 0\left|\mu_{\alpha}\right| f\right\rangle\right|^{2}
$$


where $\omega_{f}$ is excitation energy of the transition from the ground state $\langle 0|$ to the excited state $|f\rangle ; \mu_{\alpha}$ is the dipole moment operator for a certain Cartesian component $\alpha$.

Transition matrix for two-photon transition is given by the sum-over-states formula:

$$
S_{\alpha \beta}=\sum_{n} \frac{\left\langle 0\left|\mu_{\alpha}\right| n\right\rangle\left\langle n\left|\mu_{\beta}\right| f\right\rangle}{\omega_{n}-\frac{\omega_{f}}{2}}+\frac{\left\langle 0\left|\mu_{\beta}\right| n\right\rangle\left\langle n\left|\mu_{\alpha}\right| f\right\rangle}{\omega_{n}-\frac{\omega_{f}}{2}},
$$

where $\alpha, \beta$ are Cartesian coordinates $x, y, z$; summation by $n$ takes into account all states including ground and final ones. In the case of linear polarization of the incident light the transition moment $\delta^{T P A}$ averaged by all orientations of the molecules is expressed by

$$
\delta^{T P A}=\sum_{\alpha \beta}\left(S_{\alpha \alpha} S_{\beta \beta}^{*}+2 S_{\alpha \beta} S_{\alpha \beta}^{*}\right) .
$$

Two-photon absorption cross-section $\sigma^{T P A}$ describes the attenuation of the incident light with the intensity $I$ propagating along the coordinate $z$ :

$$
\frac{d I}{d z}=\frac{\sigma^{T P A}}{\omega_{i}} N_{g} I^{2}
$$

where $\omega_{i}$ is energy of an incident photon; $N_{g}$ is the number of molecules per unit volume in the ground state; it equals

$$
\sigma^{T P A}=\frac{4 \pi^{2} a_{0}^{5} \alpha}{15 c} \frac{\omega_{f}}{\Gamma} \delta^{T P A}
$$

where $a_{0}$ is the Bohr radius, $\alpha$ is the fine structure constant, $c$ is speed of light, $\Gamma$ is lifetime broadening. The value of $\Gamma$ is supposed to be $0.1 \mathrm{eV}$. The molecular two-photon cross section is usually expressed in the units of Goeppert-Mayer (GM, $1 \mathrm{GM}=10^{-50} \mathrm{~cm}^{4}$ s photon $\left.^{-1}\right)[1,3]$.

All calculations are performed with the DALTON program [4,5]. Oscillator strength and two-photon absorption cross section are obtained by linear and quadratic response TDDFT methods implemented in DALTON using the Becke three-parameter Lee-YangParr (B3LYP) functional [6] and 6-31G(d,p) basis set. The photons are supposed to have the same energy.

\section{Results and discussion}

The structure of molecules is obtained by the optimization of atom coordinates in relation to the minimum of energy using the B3LYP functional and 6-31G(d,p) basis set. The most stable conformations of the molecules are considered. The molecule of DBP can exist as two conformations which have close values of energy; they are formed by the rotation of the non-hydrogen-bonded moieties [7], the most stable structure is shown in Fig. 1c. Results of calculations for the one-photon and two-photon transitions are summarized in Table 1. The one-photon absorption spectra are dominated by the transitions to the lowest excited states $S_{1}$ and $S_{2}$ for all three compounds studied. The energy and oscillator strength of the one-photon transitions agree with the results obtained earlier [7]. The two-photon cross-section is leading for transitions to the states $\mathrm{S}_{4}$ and $\mathrm{S}_{5}$ in the case of $\mathrm{HBO}$, to $\mathrm{S}_{4}$ and $\mathrm{S}_{6}$ in the case of DBP, and to $\mathrm{S}_{3}$ in the case of BBHQ. The magnitudes of the TPA cross section of the DBP rotamer $d$ are similar to 
ones of the most stable structure $c$ except the transitions to the states $\mathrm{S}_{3}, \mathrm{~S}_{9}$, and $\mathrm{S}_{12}$ which exhibit substantially lower TPA cross sections for the rotamer $d$ than for rotamer $c$. The largest two-photon absorption cross-sections of DBP and BBHQ are about 10 times as much as the largest TPA cross section of HBO. The value for TPA cross section of $\mathrm{S}_{0}-\mathrm{S}_{3}$ transition of a BBHQ molecule is calculated to be $1520 \mathrm{GM}$. Due to the centrosymmeric structure of the molecule of BBHQ there are strict selection rules for OPA and TPA: transitions that are active in OPA are inactive in TPA and vice versa. The transition $\mathrm{S}_{0^{-}}$ $\mathrm{S}_{12}$ of a BBHQ molecule is predicted to have TPA cross section of $875 \mathrm{GM}$, however, the excitation by two photons with the same frequency requires the energy of $2.76 \mathrm{eV}$ that is near the first one-photon absorption band (the energy of the vertical transition $\mathrm{S}_{0}-\mathrm{S}_{1}$ is $2.94 \mathrm{eV}$ ). This causes small value of the denominators in the formula (2) for $\mathrm{n}=1$ but leads to the appearance of one-photon absorption.

Table 1

Calculated one-photon (OPA) and two-photon absorption (TPA) parameters for excited singlet states

\begin{tabular}{|c|c|c|c|c|c|c|}
\hline Compound & State & Symmetry & Energy, eV & $\delta^{\mathrm{OPA}}$ & $\delta^{\text {TPA }}$, a.u. & $\sigma^{\mathrm{TPA}}, \mathrm{GM}$ \\
\hline \multirow{9}{*}{$\mathrm{HBO}$} & $\mathrm{S}_{1}$ & $\mathrm{~A}^{\prime}$ & 3.92 & 0.37 & 164 & 2 \\
\hline & $\mathrm{S}_{2}$ & $\mathrm{~A}^{\prime}$ & 4.49 & 0.35 & 976 & 14 \\
\hline & $\mathrm{S}_{3}$ & $\mathrm{~A}^{\prime}$ & 4.76 & 0.03 & 305 & 5 \\
\hline & $\mathrm{S}_{4}$ & $\mathrm{~A}^{\prime}$ & 5.19 & 0.01 & 7010 & 138 \\
\hline & $\mathrm{S}_{5}$ & $\mathrm{~A}^{\prime}$ & 5.43 & 0.03 & 5550 & 120 \\
\hline & $\mathrm{S}_{6}$ & $\mathrm{~A}^{\prime \prime}$ & 5.61 & 0.00 & 2 & 0 \\
\hline & $\mathrm{S}_{7}$ & $\mathrm{~A}^{\prime}$ & 5.65 & 0.01 & 1240 & 29 \\
\hline & $\mathrm{S}_{8}$ & $\mathrm{~A}^{\prime}$ & 5.67 & 0.02 & 1070 & 25 \\
\hline & $\mathrm{S}_{9}$ & $\mathrm{~A}^{\prime}$ & 5.86 & 0.06 & 85 & 2 \\
\hline \multirow{12}{*}{$\begin{array}{c}\mathrm{DBP} \\
\text { rotamer c / } \\
\text { rotamer d }\end{array}$} & $\mathrm{S}_{1}$ & $\mathrm{~A}^{\prime}$ & $3.44 / 3.46$ & $1.13 / 1.14$ & $199 / 269$ & $2 / 2$ \\
\hline & $\mathrm{S}_{2}$ & $\mathrm{~A}^{\prime}$ & $3.86 / 3.80$ & $0.30 / 0.27$ & $12200 / 12000$ & 134 / 127 \\
\hline & $\mathrm{S}_{3}$ & $\mathrm{~A}^{\prime}$ & $4.15 / 4.17$ & $0.01 / 0.02$ & $10400 / 269$ & $131 / 3$ \\
\hline & $\mathrm{S}_{4}$ & $\mathrm{~A}^{\prime}$ & $4.28 / 4.28$ & $0.03 / 0.03$ & $86400 / 94100$ & $1160 / 1260$ \\
\hline & $\mathrm{S}_{5}$ & $\mathrm{~A}^{\prime}$ & $4.40 / 4.40$ & $0.01 / 0.01$ & $451 / 418$ & $6 / 6$ \\
\hline & $\mathrm{S}_{6}$ & $\mathrm{~A}^{\prime}$ & $4.76 / 4.78$ & $0.01 / 0.02$ & $25300 / 24900$ & $420 / 418$ \\
\hline & $\mathrm{S}_{7}$ & $\mathrm{~A}^{\prime \prime}$ & $4.95 / 4.93$ & $0.00 / 0.00$ & $8 / 8$ & $0 / 0$ \\
\hline & $\mathrm{S}_{8}$ & $\mathrm{~A}^{\prime}$ & $5.10 / 5.06$ & $0.05 / 0.11$ & $2610 / 4720$ & $50 / 88$ \\
\hline & $\mathrm{S}_{9}$ & $\mathrm{~A}^{\prime}$ & $5.16 / 5.16$ & $0.02 / 0.00$ & $1120 / 335$ & $22 / 7$ \\
\hline & $\mathrm{S}_{10}$ & $\mathrm{~A}^{\prime}$ & $5.25 / 5.25$ & $0.03 / 0.04$ & $1160 / 1080$ & $24 / 22$ \\
\hline & $\mathrm{S}_{11}$ & $\mathrm{~A}^{\prime \prime}$ & $5.35 / 5.33$ & $0.00 / 0.00$ & $9 / 7$ & $0 / 0$ \\
\hline & $\mathrm{S}_{12}$ & $\mathrm{~A}^{\prime}$ & $5.36 / 5.32$ & $0.01 / 0.00$ & $509 / 113$ & $11 / 2$ \\
\hline \multirow{15}{*}{ BBHQ } & $\mathrm{S}_{1}$ & $\mathrm{~B}_{\mathrm{u}}$ & 2.94 & 0.46 & 0 & 0 \\
\hline & $\mathrm{S}_{2}$ & $\mathrm{~B}_{\mathrm{u}}$ & 3.76 & 0.98 & 0 & 0 \\
\hline & $\mathrm{S}_{3}$ & $A_{g}$ & 4.12 & 0.00 & 122000 & 1520 \\
\hline & $\mathrm{S}_{4}$ & $\mathrm{~A}_{\mathrm{g}}$ & 4.30 & 0.00 & 7330 & 99.2 \\
\hline & $\mathrm{S}_{5}$ & $\mathrm{~B}_{\mathrm{u}}$ & 4.31 & 0.02 & 0 & 0 \\
\hline & $\mathrm{S}_{6}$ & $A_{g}$ & 4.32 & 0.00 & 1170 & 16 \\
\hline & $\mathrm{S}_{7}$ & $\mathrm{~B}_{\mathrm{u}}$ & 4.77 & 0.01 & 0 & 0 \\
\hline & $\mathrm{S}_{8}$ & $\mathrm{~A}_{\mathrm{g}}$ & 4.92 & 0.00 & 985 & 17.5 \\
\hline & $\mathrm{S}_{9}$ & $A_{g}$ & 5.05 & 0.00 & 8910 & 167 \\
\hline & $\mathrm{S}_{10}$ & $\mathrm{~B}_{\mathrm{u}}$ & 5.11 & 0.01 & 0 & 0 \\
\hline & $\mathrm{S}_{11}$ & $A_{u}$ & 5.20 & 0.00 & 0 & 0 \\
\hline & $\mathrm{S}_{12}$ & $\mathrm{~B}_{\mathrm{g}}$ & 5.30 & 0.00 & 55 & 1 \\
\hline & $\mathrm{S}_{13}$ & $\mathrm{~B}_{\mathrm{u}}$ & 5.30 & 0.03 & 0 & 0 \\
\hline & $\mathrm{S}_{14}$ & $\mathrm{~B}_{\mathrm{u}}$ & 5.36 & 0.06 & 0 & 0 \\
\hline & $\mathrm{S}_{15}$ & $A_{g}$ & 5.53 & 0.00 & 39100 & 875 \\
\hline
\end{tabular}

The excitation energy for the transitions with the largest TPA cross section of HBO and BBHQ is close to the energy of vertical transitions for the keto structures in the 
excited states which correspond to the maxima of fluorescence bands with an anomalously large Stokes shift. The calculated values for the vertical transitions of the keto structures reported in [7] are $2.67 \mathrm{eV}$ for $\mathrm{HBO}$ and $1.97 \mathrm{eV}$ for BBHQ. The corresponding values of excitation energy $\omega_{f} / 2$ are 2.60 and $2.06 \mathrm{eV}$. In the case of DBP the energies of excitation and fluorescence transition are $2.66 \mathrm{eV} \mathrm{[7]} \mathrm{and} 2.14 \mathrm{eV}$.

\section{Conclusions}

Theoretical modeling of two-photon absorption is performed for three benzoxazoles exhibiting excited state intramolecular proton transfer. Molecules that possess two beznoxazole fragments are predicted to be more efficient in two-photon absorption than 2-(2'-hydroxyphenyl)benzoxazole. Transitions with large two-photon absorption cross section have higher energy than the lowest transitions that demonstrate large oscillator strength for one-photon absorption. Nevertheless, it is possible to excite these transitions by the two-photon absorption with photons, which energy is substantially below the edge of the one-photon absorption.

\section{References}

1. Hales, J.M. Two-photon absorption: concepts, molecular materials and applications / J.M. Hales, S.-H. Chi, V.W. Chen, J.W. Perry // Chapter 13 in: The WSPC Reference on Organic Electronics: Organic Semiconductors. - World Scientific. - 2016. - P. $397-441$.

2. Shang, $\mathbf{X}$. Optical nonlinearities and transient dynamics of 2-(2'hydroxyphenyl)benzoxazole studied by single-beam and time-resolved two-color Z-scan techniques / X. Shang, G. Tang, G. Zhang, Y. Liu, W. Chen, B Yang. X. Zhang // J. Opt. Soc. Am. B. - 1998. - Vol. 15. - P. $854-862$.

3. Salem, M.A. Two-Photon Absorption in Fluorescent Protein Chromophores: TDDFT and CC2 Results / M.A. Salem, A. Brown // J. Chem. Theory Comput. - 2014. Vol. 10. - P. 3260 - 3269.

4. Aidas, K. The Dalton quantum chemistry program / K. Aidas, C. Angeli, K.L. Bak, V. Bakken, R. Bast, L. Boman, O. Christiansen, R. Cimiraglia, S. Coriani, P. Dahle, E. K. Dalskov, U. Ekstroem, T. Enevoldsen, J. J. Eriksen, P. Ettenhuber, B. Fernandez, L. Ferrighi, H. Fliegl, L. Frediani, K. Hald, A. Halkier, C. Haettig, H. Heiberg, T. Helgaker, A.C. Hennum, H. Hettema, E. Hjertenaes, S. Hoest, I.-M. Hoeyvik, M.F. Iozzi, B. Jansik, H.J. Aa. Jensen, D. Jonsson, P. Joergensen, J. Kauczor, S. Kirpekar, T. Kjaergaard, W. Klopper, S. Knecht, R. Kobayashi, H. Koch, J. Kongsted, A. Krapp, K. Kristensen, A. Ligabue, O.B. Lutnaes, J.I. Melo, K.V. Mikkelsen, R.H. Myhre, C. Neiss, C.B. Nielsen, P. Norman, J. Olsen, J.M.H. Olsen, A. Osted, M.J. Packer, F. Pawlowski, T.B. Pedersen, P.F. Provasi, S. Reine, Z. Rinkevicius, T.A. Ruden, K. Ruud, V. Rybkin, P. Salek, C.C. M. Samson, A. Sanchez de Meras, T. Saue, S.P.A. Sauer, B. Schimmelpfennig, K. Sneskov, A.H. Steindal, K.O. Sylvester-Hvid, P.R. Taylor, A.M. Teale, E.I. Tellgren, D. P. Tew, A.J. Thorvaldsen, L. Thoegersen, O. Vahtras, M.A. Watson, D.J.D. Wilson, M. Ziolkowski, H. Agren, // WIREs Comput. Mol. Sci. - 2014. - Vol. 4. - P. 269 - 284.

5. Dalton, a Molecular Electronic Structure Program. Release Dalton2017.alpha (2017), http://daltonprogram.org.

6. Becke, A.D. Density-functional thermochemistry. III. The role of exact exchange / A.D. Becke // J. Chem. Phys. - 1993. - Vol. 98. - P. 5648 - 5652.

7. Syetov, Y. TDDFT Calculations of Electronic Spectra of Benzoxazoles Undergoing Excited State Proton Transfer / Y. Syetov // J. Fluoresc. - 2013. - Vol. 23. P. $689-696$. 